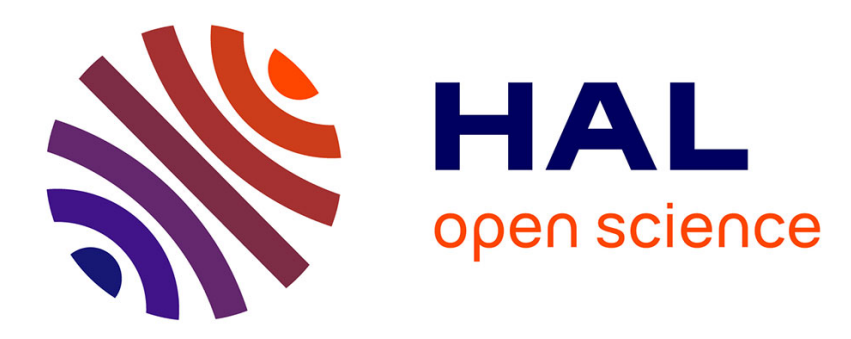

\title{
Évaluation et contrôle des interfaces dans les hétéro-structures silicium/germanium
}

J.-M. Baribeau

\section{To cite this version:}

J.-M. Baribeau. Évaluation et contrôle des interfaces dans les hétéro-structures silicium/germanium. Journal de Physique IV Proceedings, 1997, 07 (C6), pp.C6-91-C6-100. 10.1051/jp4:1997608 . jpa00255707

\section{HAL Id: jpa-00255707 https://hal.science/jpa-00255707}

Submitted on 1 Jan 1997

HAL is a multi-disciplinary open access archive for the deposit and dissemination of scientific research documents, whether they are published or not. The documents may come from teaching and research institutions in France or abroad, or from public or private research centers.
L'archive ouverte pluridisciplinaire HAL, est destinée au dépôt et à la diffusion de documents scientifiques de niveau recherche, publiés ou non, émanant des établissements d'enseignement et de recherche français ou étrangers, des laboratoires publics ou privés. 


\title{
Évaluation et contrôle des interfaces dans les hétéro-structures silicium/germanium
}

\author{
J.-M. Baribeau \\ Institut des Sciences des Microstructures, Conseil National de Recherches du Canada, Ottawa, K1A 0R6, \\ Canada
}

\begin{abstract}
Résumé : L'utilisation des alliages $\mathrm{Si}_{1-\mathrm{x}} \mathrm{Ge}_{\mathrm{X}}$ dans les dispositifs électroniques et opto-électroniques connait un essor considérable en raison des degrés de liberté supplémentaires qu'elle offre dans la conception et l'optimisation des dipositifs. L'adjonction du silicium et des alliages $\mathrm{Si}_{1-\mathbf{x}} \mathrm{Ge}_{\mathbf{x}}$ présente toutefois des difficultés reliées à la gestion des contraintes et au contrôle de la morphologie et de la composition des hétéro-structures. Nous avons appliqué les méthodes de diffusion des rayons-x à l'évaluation des interfaces d'hétéro-structures $\mathrm{Si} / \mathrm{Si}_{1}-\mathrm{x} \mathrm{Ge}_{\mathrm{x}}$ préparées par épitaxie par jets moléculaires. Pour les systèmes comportant des couches d'alliages dilués (i.e., $\mathrm{x}<0.35$ ) on observe des hétéro-interfaces planes pour des dépôts à basse température $\left(500^{\circ} \mathrm{C}\right)$ mais une ondulation marquée des interfaces de films déposés à haute température $\left(>625^{\circ} \mathrm{C}\right)$. Ce changement morphologique s'explique par la minimisation de l'énergie associée à la contrainte aux dépens de l'énergie libre de surface. Dans le cas d'alliages riches en $\mathrm{Ge}(\mathrm{x} \sim 1.0)$ la température de croissance et la désorientation résiduelle du substrat ont une incidence marquée sur l'amplitude de la rugosité d'interface et sur sa distribution spatiale. Dans les multi-couches Ge/Si on observe la présence de traces de Ge dans les couches de Si causée par une ségrégation de la couche de Ge sousjacente. Ce phénomène peut-être minimisé par une exposition de la surface à l'hydrogène atomique durant le dépôt.
\end{abstract}

Summary : Use of $\mathrm{Si}_{1-x} \mathrm{Ge}_{\mathrm{x}}$ alloys in electronic devices is expanding rapidly because of the additional degrees of freedom it allows in the design and optimisation of components. Combining $\mathrm{Si}$ with $\mathrm{Si}_{1-\mathrm{x}} \mathrm{Ge}_{\mathrm{X}}$ alloys is made difficult because of problems associated to the control of strain, morphology and compositional uniformity in the heterostructures. $\mathrm{X}$-ray scattering techniques have been used to assess the interfaces in various $\mathrm{Si} / \mathrm{Si}_{1} \mathrm{x}_{\mathrm{X}} \mathrm{Ge}_{\mathrm{X}}$ heterostructures prepared by molecular beam epitaxy. In structures comprising dilute alloys (i.e., $x<0.35$ ) planar interfaces are observed at low growth temperature $\left(500^{\circ} \mathrm{C}\right)$ but wavy interfaces are observed in films deposited at high temperature $\left(>625^{\circ} \mathrm{C}\right)$. This morphological evolution is explained by the minimization of the strain energy to the expense of surface free energy. In the case of structures madé of thin Ge-rich $(x \sim 1.0)$ layers, the growth temperature and the residual substrate misorientation determine the amplitude of the interface roughness and its spatial distribution. In thin $\mathrm{Ge} / \mathrm{Si}$ heterostructures traces de Ge are observed in the Si cap due to Ge surface segregation. This effect is much reduced by exposing the surface during growth to a flux of atomic hydrogen.

\section{INTRODUCTION}

L'utilisation des alliages $\mathrm{Si}_{1-\mathrm{x}} \mathrm{Ge}_{\mathrm{X}}$ dans les dispositifs électroniques et opto-électroniques connait un essor considérable [1]. L'ajout de germanium amène deux avantages de taille. D'une part, la différence de bande interdite entre le silicium et l'alliage $\mathrm{Si}_{1-\mathrm{x}} \mathrm{Ge}_{\mathrm{x}}$ résulte en une discontinuité de bandes aux hétéro-jonctions. Ce phénomène peut être mis à profit pour l'amélioration des caractéristiques des dispositifs. Le meilleur exemple est sans nul doute l'amélioration des performances à hautes fréquences qu'apporte l'utilisation d'alliages $\mathrm{Si}_{1-\mathrm{x}} \mathrm{Ge}_{\mathrm{X}}$ dans la base de transistors bipolaires [2]. D'autre part, le désaccord de maille entre le silicium et l'alliage $\mathrm{Si}_{1-\mathrm{x}} \mathrm{Ge}_{\mathrm{x}}$ induit une déformation élastique du réseau qui influe également sur les propriétés électriques du matériau. L'utilisation du $\mathrm{Si}_{1-\mathrm{x}} \mathrm{Ge}_{\mathrm{x}}$ offre donc des degrés de liberté supplémentaires dans la conception des dipositifs et permet une meilleure optimisation des performances de ceux-ci.

L'adjonction du silicium et des alliages $\mathrm{Si}_{1-\mathrm{x}} \mathrm{Ge}_{\mathrm{x}}$ n'est pas sans présenter de difficultés. Par exemple, la contrainte élastique impose une limite dans l'épaisseur d'une couche d'alliage qui peut-être déposée sur le silicium sans introduction de défauts structuraux. De plus, cette même contrainte peut entrainer une croissance non-plane (tridemensionnelle) également indésirable dans les dispositifs. Enfin, des phénomènes d'échanges atomiques (inter-diffusion, ségrégation) au niveau des interfaces peuvent avoir un effet néfaste sur les performances des dispositifs.

Nous avons appliqué les méthodes de diffusion des rayons-x à l'évaluation des interfaces dans diverses hétéro-structures $\mathrm{Si} / \mathrm{Si}_{1-\mathrm{x}} \mathrm{Ge}_{\mathrm{x}}$ préparées par épitaxie par jets moléculaires. Pour les systèmes 
comportant des couches d'alliages diluès $(1 . e ., \mathrm{x}<0.35)$ on observe des hetèro-intertaces planes pour des dépôts à basse température $\left(500^{\circ} \mathrm{C}\right)$. On note toutefois une ondulation marquée des interfaces de films déposés à haute température $\left(>625^{\circ} \mathrm{C}\right)$. Ce changement morphologique s'explique par la minimisation de l'énergie associée à la contrainte aux dépens de l'énergie libre de surface. Dans le cas d'alliages riches en Ge (i.e., $x$ voisin de 1.0) la température de croissance et la désorientation résiduelle du substrat ont une incidence marquée sur l'amplitude de la rugosité d'interface et sur son spectre de fréquences spatiales. L'influence du substrat sur la structure des interfaces offre des perspectives intéressantes en vue de la réalisation de nano-structures ordonnées. Dans les multi-couches $\mathrm{Si} / \mathrm{Ge}$ on observe la présence de traces de $\mathrm{Ge}$ dans les couches de Si causée par une ségrégation de la couche de Ge sous-jacente. Ce phénomène peut-être minimisé par une exposition de la surface à l'hydrogène atomique durant le dépôt. Dans cetravail nous ferons un survol de récents développements dans l'étude de ces questions.

\section{DÉTAILS EXPÉRIMENTAUX}

\subsection{Croissance épitaxiale}

La méthodologie utilisée pour la croissance des diverses hétéro-structures est décrite en détail ailleurs [3-4]. Brièvement, les dépôts sont réalisés par épitaxie par jets moléculaires au moyen d'un réacteur V80 de VG Semicon. Les dépôts, effectués sous vide poussé $\left(<10^{-7} \mathrm{~Pa}\right)$, ont été réalisés sur plaquettes de Si (001) de $100 \mathrm{~mm}$ légèrement dôpées (résistivité de 5-50 $\Omega-\mathrm{cm}$ ). La température du substrat est mesuréc par pyrométrie infra-rouge et les taux de déposition (typiquement de $0.1 \mathrm{~nm} / \mathrm{s}$ ) contrôlés par spectroscopie de l'émission induite par impact électronique (système Sentinel III). Les jets d'hydrogène atomique sont obtenus par dissociation de l'hydrogène moléculaire au contact d'un filament de tungstène porté à haute température (cellule commerciale de EPI MBE Products Group). Typiquement, une température de filament de $2150^{\circ} \mathrm{C}$ et un débit d'hydrogène moléculaire de $\sim 10^{-3} \mathrm{~Pa}$ est utilisé. On estime le rendement dissociatif à environ $3-5 \%$ dans ces conditions [5].

\subsection{Mesures de diffusion rayons-x}

Plusieurs types de mesures sont présentés. Un diffractomètre Philips MRD (radiation $\mathrm{Cu} \mathrm{K}_{\alpha}$ ) muni d'un monochromateur du type Bartel (utilisé en mode 220, il possède une résolution angulaire de $12 \mathrm{sec}$ ) est utilisé pour les mesures de courbes oscillantes au voisinage d'un faisceau diffracté. Des mesures de distribution d'intensité dans l'espace reciproque sont réalisées en insérant une fente de $0.1 \mathrm{~mm}$ devant le détecteur. En diffraction, la réflexion 113 avec angle de sortie rasant est sélectionnée en raison de la meilleure résolution angulaire qu'offre cette géométrie [6]. Des mesures de réflectivité hors-spéculaires ont également été réalisées dans cette configuration.

Des mesures de réflectivité en incidence rasante sont réalisées au moyen d'un goniomètre Philips 1820 avec une divergence du faisceau incident de $0.25^{\circ}$ et une résolution angluaire de $0.015^{\circ}$. Ce système permet d'effectuer des balayages dans la direction spéculaire par une rotation coordonnée de l'angle d'incidence et de détection et des mesures hors-spéculaires en découplant ces axes de rotation. En particulier, des mesures de profil d'intensité en géométrie transversale ont été obtenues en réduisant la divergence angulaire à $0.1^{\circ}$ par l'insertion d'une fente à l'entré du goniomètre. Les profils de réflexion spéculaire sont modélisés au moyen de la méthode récursive de Parratt [7] alors que l'intensité horsspéculaire en géométrie transversale est calculée dans l'approximation de Born avec ondes déformées (distorted wave Born approximation) [8].

\section{RÉSULTATS EXPÉRIMENTAUX ET DISCUSSION}

\subsection{Changements morphologiques des alliages $\mathrm{Si}_{1-\mathrm{x}} \mathrm{Ge}_{\mathrm{x}}$ contraints}

Asaro et Tiller [9] et Grinfeld [10] ont montré que la surface d'un solide soumis à un stress était instable à la formation d'ondulations. En effet, la réduction d'énergie associée au relâchement du stress par l'intermédiaire de ce changement morphologique excède le gain d'énergie de surface associé aux ondulations. Dans le cas de l'épitaxie sous contrainte, on s'attend à ce qu'un dépôt présentant initialement une morphologie plane ait tendance à devenir rugueux à mesure que l'énergie associée à la contrainte 

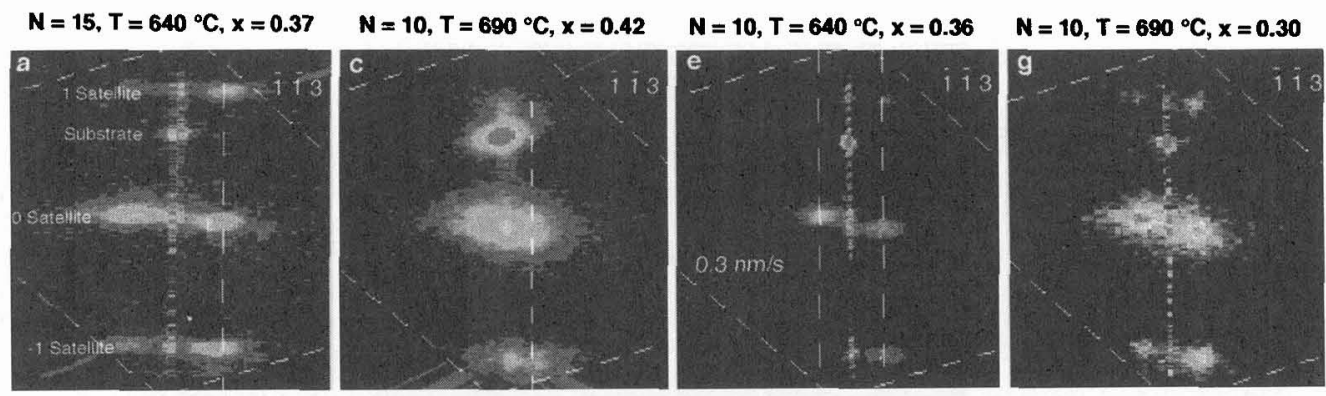

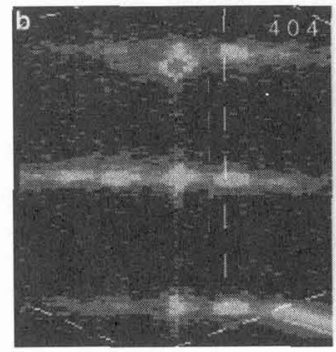

$N=15, T=640^{\circ} \mathrm{C}, \mathrm{x}=0.37$

$0.2 \mathrm{~nm}^{-1}$

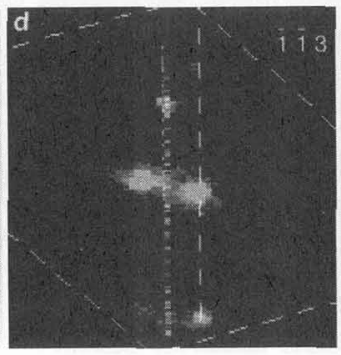

$N=10, T=690^{\circ} \mathrm{C}, \mathrm{x}=0.30$

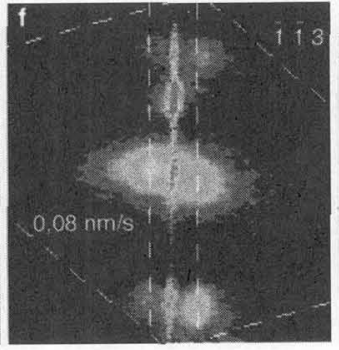

$N=10, T=640^{\circ} \mathrm{C}, \mathrm{x}=0.36$

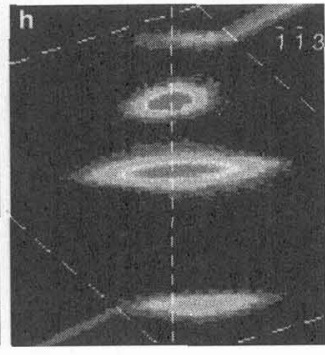

$\mathrm{N}=40, \mathrm{~T}=690^{\circ} \mathrm{C}, \mathrm{x}=0.30$

Figure 1: Intensité diffractée (échelle logarithmique) autour du pic de Bragg $\overline{1} \overline{1} 3$ et $\overline{4} 0 \overline{4}$ dans l'espace réciproque (q $q_{\perp}$ vs $q_{/ /}$) pour différents super-réseaux $\mathrm{Si} / \mathrm{Si}_{1-\mathrm{X}} \mathrm{Ge}_{\mathrm{X}}$. Le nombre de périodes $\mathrm{N}$, la température du dépôt $\mathrm{T}$ et la concentration de germanium $\mathrm{x}$ sont indiqués à chaque panneau. Les losanges hachurés délimitent la zone balayée dans l'expérience; l'intensité hors de cette zone provient d'erreurs d'interpolation et est sans signification. Dans ces cartes l'échelle verticale est aussi multipliée par un facteur 0.5 pour mieux apprécier les lobes latéraux.

(i.e., l'épaisseur) augmente et, qu'éventuellement, des ondulations de surface de longueur d'onde bien définie se développent.

Le phénomène d'ondulation de surface est assez bien documenté sur les systèmes tels que $\mathrm{Si}_{1}$. ${ }_{\mathrm{x}} \mathrm{Ge}_{\mathrm{x}} / \mathrm{Si}$ [11] et InGaAs/GaAs [12]. On dispose toutefois d'assez peu de données dans le cas des interfaces contraintes dans les super-réseaux et on sait peu sur l'influence de paramètres expérimentaux tels que la vitesse et température de croissance sur la formation de ces ondulations. Nous avons appliqué la méthode de "cartographie" de l'intensité diffractée à l'étude des ondulations d'interface sur plusieurs hétérostructures $\mathrm{Si}_{1-\mathrm{x}} \mathrm{Ge}_{\mathrm{x}} / \mathrm{Si}$. Les structures examinées sont constituées d'un empilement (typiquement 20 couches) où alternent des couches de $\mathrm{Si}$ et de $\mathrm{Si}_{1-\mathrm{x}} \mathrm{Ge}_{\mathrm{x}}$ (x voisin de 0.35 ) de 10 et $5 \mathrm{~nm}$ d'épaisseur, respectivement. La Figure 1 présente une série de cartes montrant l'intensité diffractée au voisinage du faisceau 113 (et 404.) pour différents échantillons. Dans ces cartes on observe une tache de forte intensité associée au substrat de même que trois pics satellites dus à la super-périodicité des couches déposés (cf. Fig. 1 (a)) Ces pics sont alịgnés verticalement (même valeur de $q / /$ ) avec le pic du substrat indiquant l'absence de relaxation des contraintes dans le super-réseau. Ces cartes révèlent aussi la présence de lobes secondaires de chaque côté des pics satellites. Ces lobes indiquent la présence d'une modulation latérale des interfaces. Ce phénomène est observé à des températures de dépôt supérieures à $\sim 625^{\circ} \mathrm{C}$. La longueur d'onde des ondulations donnée par $\Lambda=2 \pi / \Delta q / /$, où $\Delta q / /$ est la position relative du lobe secondaire, est typiquement de $100 \mathrm{~nm}$ à $690^{\circ} \mathrm{C}$ et augmente jusqu'à $\sim 200 \mathrm{~nm}$ à $745^{\circ} \mathrm{C}$.

L'intensité et le caractère des lobes latéraux varient en fonction des conditions de croissance et des paramètres du super-réseau. Une comparaison de cartes 113 et 404 obtenues sur un même échantillon (Fig. 1 (a) et 1 (b)) montre que ces lobes sont plus accentués et plus éloignés dans le cas du pic de diffraction d'indice 404. Des lobes de deuxième et troisième ordre sont aussi visibles dans cette dernière géométrie. Ceci indiaue une modulation d'interface orientée préférentiellement selon une direction cristal- 
(a)

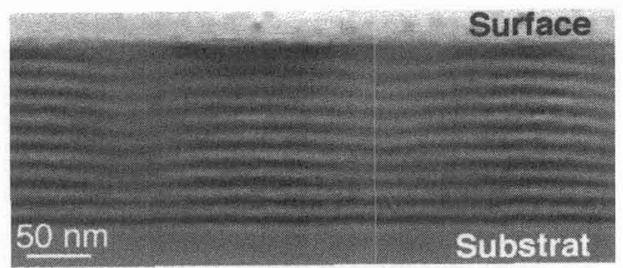

(b)

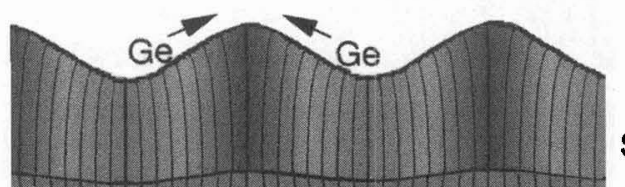

$\mathrm{SiGe}$

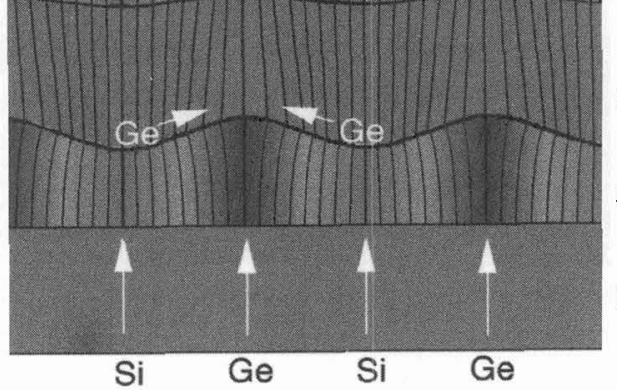

$\mathrm{Si}$

SiGe

Si (001)

(c)

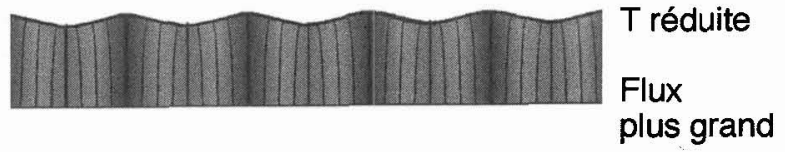

Figure 2: (a) Coupe transversale obtenue par microscopie électronique par transmission d'un super-réseau $\mathrm{Si}_{0.65} \mathrm{Ge} 0.35 / \mathrm{Si}$ préparé à $750^{\circ} \mathrm{C}$. Les ondulations des interfaces sont clairement visibles. (b) Vue schématique des ondulations des interfaces. (c) Effets d'une réduction de température ou d'une augmentation de la vitesse de dépôt sur la longueur d'onde des ondulations aux interfaces.

lographique [100]. Une augmentation de la teneur en Ge des alliages, pour sa part, cause un plus grand étalement des lobes secondaires (Fig. 1 (c) et 1 (d)) sans toutefois en modifier l'écart du satellite principal. Ce dernier phénomène est toutefois observé en changeant la vitesse de croissance pour une structure donnée (Fig. 1 (e) et 1 (f)). Enfín, une augmentation de l'épaisseur totale du super-réseau conduit ultimement à une relaxation des contraintes qui ce traduit par une modification marquée du profil d'intensité des satellites (Fig. 1 (h)). Les pics satellites ne sont alors plus alignés verticalement avec la réflexion du substrat et présentent la forme elliptique caractéristique d'une structure maclée.

La Figure 2 (a) montre un cliché de microscopie électronique en coupe transversale d'un super-réseau préparé à une température de $750^{\circ} \mathrm{C}$. Les ondulations des interfaces, clairement visibles par microscopie, sont représentées schématiquement à la Figure 2 (b). Les couches d'alliage croissent de manière nonplane comme en témoigne la forte ondulatiơn aux interfaces $\mathrm{Si}_{1-\mathrm{x}} \mathrm{Ge}_{\mathrm{X}} / \mathrm{Si}$. L'interface recouvre en partie sa morphologie plane par l'insertion d'une couche de Si. La planéité n'est toutefois pas parfaite et de nouvellês ondulations alignées avec celles sous-jacentes apparaissent lorsqu'une couche additionnelle d'alliage est déposée. Le contraste du cliché de microscopie suggère également une concentration accrue de Ge au maximum des ondulations. Le scénario qui se dégage de cette analyse est le suivant. A haute température, la diffusion atomique de surface devient appréciable et les atomes de Ge tendent à s'agglomérer en ilôts tridimensionnels où le pas de réseau est mieux adapté. Les régions pauvres en $\mathrm{Ge}$ pour leur part adoptent une pas de réseau voisin de celui du Si. En dépit de l'augmentation d'énergie de surface, cette configuration se traduit par une diminution globale de l'énergie à cause de la réduction de 


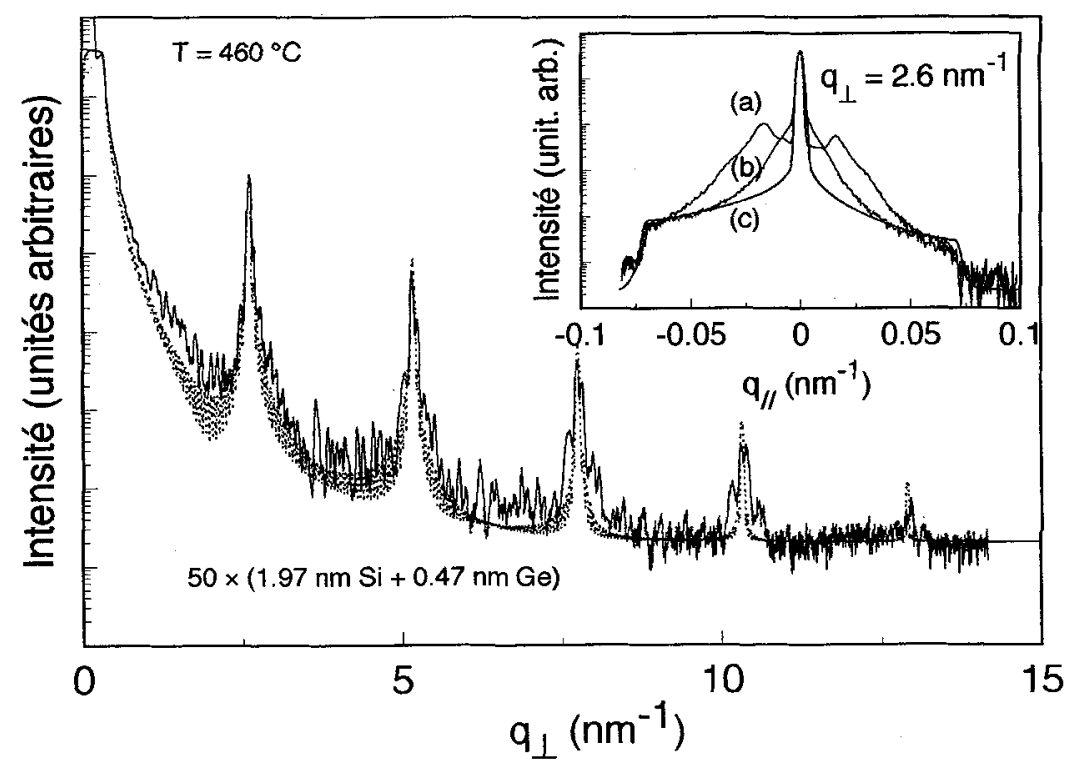

Figure 3: Réflexion des rayons-x dans la direction spéculaire pour un super-réseau $\mathrm{Si} / \mathrm{Ge}$. La courbe en pointillés est le résultat d'une simulation effectuée au moyen du modèle récursif de Parratt [6] et inclue une rugosité d'interface de type DebyeWaller [12]. L'encart montre le profil du premier satellite mesuré par un balayage transversal (a) parallèle à la direction de désorientation $\left(0.48^{\circ}\right)$ du substrat et perpendiculaire (b) à cette direction. La courbe (c) est un calcul effectué dans l'approximation de Born modifiée en utilisant les paramètres de rugosité déterminés dans la mesure spéculaire.

la déformation moyenne du réseau dans la direction parallèle à la surface. Des modèles théoriques récents prédisent une telle décomposition latérale des alliages sous contraites [[13-14].

L'amplitude et la longueur d'onde des ondulations peuvent être contrôlées dans une certaine mesure par la température et la vitesse du dépôt (Fig. 2 (c)). Dans le cas d'un super-réseau, on s'attend par ailleurs à ce que l'amplitude des ondulations et leur corrélation verticale puissent être contrôlées par l'épaisseur des couches de $\mathrm{Si}$ intercalées. On remarque en effet que le Si doit être contraint localement au sommet des îlots pour s'adapter au pas de l'alliage ce qui favorise l'alignement des îlots des couches d'alliage subséquentes. Pour la géométrie étudiée ici, l'épaisseur des couches de Si est suffisante pour préserver la corrélation verticale mais trop grande pour le développement d'une discontinuité des couches d'alliage et la formation d'îlots discrets. Un tel phénomène, observé récemment [15], offre des perspectives intéressantes en vue de fabriquation de points quantiques ordonnés.

\subsection{Interfaces dans les super-réseaux $\mathrm{Si} / \mathrm{Ge}$}

Il y a actuellement un vaste intérêt dans l'étude de la rugosité des interfaces dans les multi-couches semiconductrices. La réduction constante des dimensions des dispositifs exige en effet une connaissance précise de l'évolution spatiale (dans le plan de l'interface) et temporelle (en fonction de l'épaisseur) de la rugosité de même qu'une bonne compréhension des phénomènes physiques qui régissent cette évolution. Les super-réseaux Si/Ge où alternent des couches de Si et de Ge pures d'une épaisseur de quelques monocouches constituent des systèmes contraints de dimensions verticales réduites idéaux à l'étude de ces questions. Nous présentons ici quelques résultats d'une comparaison des interfaces de divers superréseaux $\mathrm{Si} / \mathrm{Ge}$ obtenus par réflectivité des rayons- $\mathrm{x}$ à incidence rasante.

La Figure 3 montre la courbe de réflectivité dans la direction spéculaire (où $\mathrm{q}_{\perp}=4 \frac{\pi}{\lambda} \sin \theta$ ) d'un superréseau $\mathrm{Si} / \mathrm{Ge}$ de 50 périodes. On y distingue des pics satellites associés à une super-périodicité de $\sim 2.5$ $\mathrm{nm}$. En plus des pics satellites, la courbe mesurée présente des oscillations sporadiques qui sont dues aux 


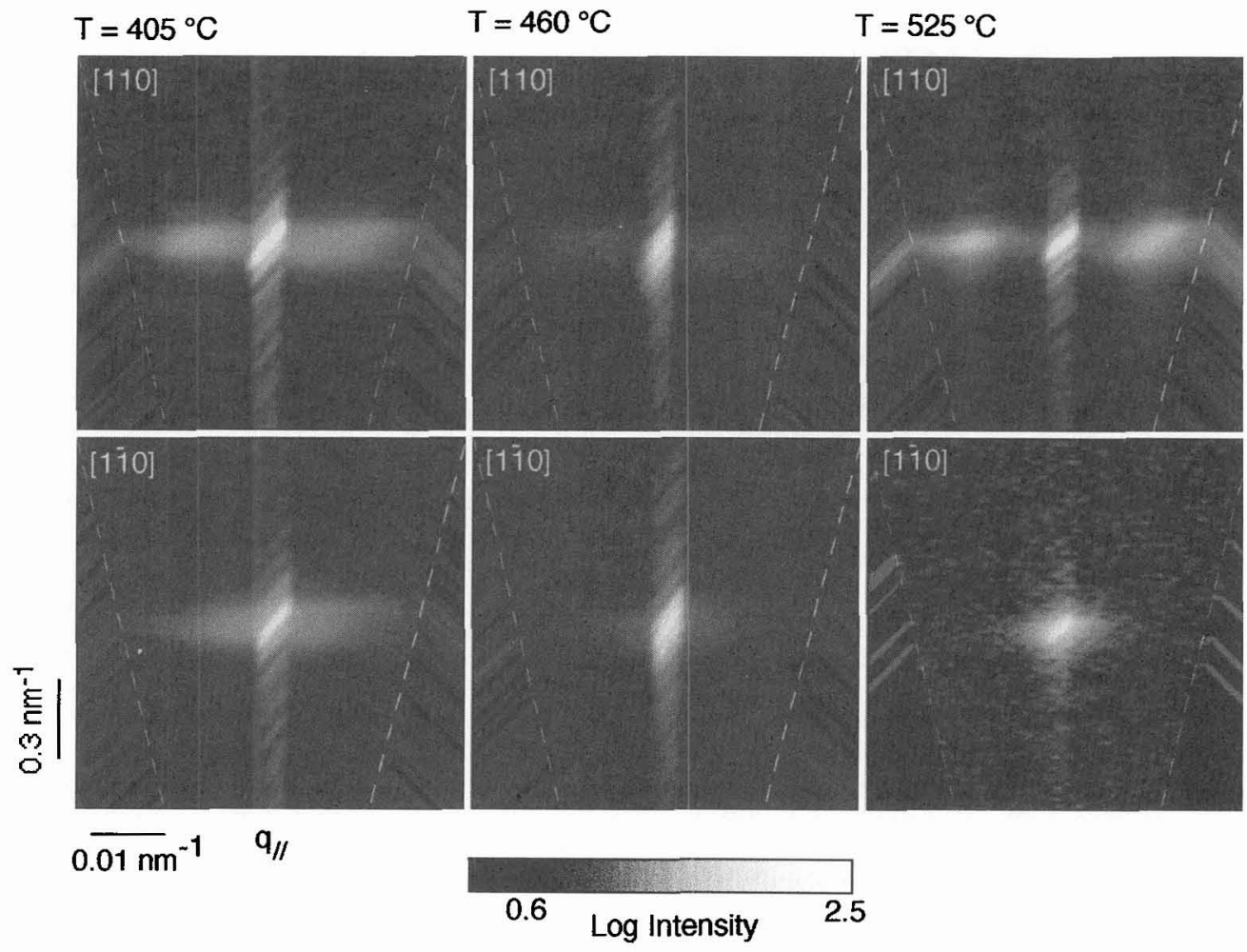

Figure 4: Carte d'intensité (échelle logarithmique) dans l'espace réciproque ( $q_{\perp}$ vs $q_{/ \prime}$ ) mesurée autour de la direction spéculaire au voisinage du premier pic satellite (i.e., $\mathrm{q}_{\perp} \sim 5 \mathrm{~nm}^{-1}$ ) pour trois super-réseaux $\mathrm{Si} / \mathrm{Ge}$ préparés à la température indiquée. Les lignes obliques hachurées délimitent la zone balayée dans l'expérience; l'intensité hors de cette zone provient d'erreurs d'interpolation et est sans signification. Notez que les échelles verticales et horizontales sont différentes dans ces cartes. La désorientation du substrat est dans la direction [110] et a pour valeur $0.40^{\circ}, 0.40^{\circ}$ et $0.48^{\circ}$ pour les températures de 405,460 et $525^{\circ} \mathrm{C}$, respectivement.

fluctuations aléatoires de l'épaisseur des couches du super-réseau [16]: A l'exception de ces pics irréguliers, impossibles à décrire précisément, le profil expérimental est bien reproduit par un modèle comportant des couches de $\mathrm{Si}$ et de Ge de 1.97 et $0.47 \mathrm{~nm}$ d'épaisseur et possédant une rugosité d'interface $\sigma$ de 0.17 et $0.27 \mathrm{~nm}$, respectivement. Notons toutefois que l'intensité intégrée des pics satellites d'ordre élevé est supérieure à celle calculée. Ceci s'explique par la réflexion diffuse dans la direction spéculaire qui devient non-négligeable aux grandes valeurs de $\mathrm{q}_{\perp}$.

L'encart de la Figure 3 montre une mesure d'intensité en géométrie transversale obtenue à la valeur de $q_{\perp}$ correspondant au premier satellite. On y remarque une faible composante diffuse reliée à la rugosité des interfaces. L'intensité hors-spéculaire varie en fonction de l'angle azimutal comme le montre la comparaison d'une mesure effectuée dans un plan d'incidence parallèle (courbe (a) ) ou perpendiculaire (courbe (b)) à la direction de la désorientation du substrat. La présence de lobes secondaires dans cette dernière géométrie suggère l'existence d'une modulation des interfaces d'une longueur d'onde $\Lambda$ bien définie. Celle-ci, donnée par $\Lambda=2 \pi / \Delta q / /$, où $\Delta q / /$ est la position du lobe secondaire, vaut environ $400 \mathrm{~nm}$ pour cet échantillon soit environ 20 fois la distance moyenne entre les marches si on suppose que la désorientation (ici $0.48^{\circ}$ selon la direction [110]) est assumée par des marches mono-atomiques.

L'intensité diffusée aux grandes valeurs de q// est assez bien reproduite par un modèle qui utilise une fonction de corrélation de la forme [17] $\mathrm{C}(\mathrm{X})=\sigma^{2} \exp -(\mathrm{X} / \xi)^{2 \mathrm{~h}}$ où $\xi$ est la longueur de corrélation dans le plan de l'interface et l'exposant $\mathrm{h}$ détermine le spectre en fréquence spatiale de la rugosité. La courbe (c) est obtenue avec les valeurs de $\sigma$ données plus haut en prenant $\xi=200 \mathrm{~nm}$ et $h=0.35$. Le désaccord aux 
petıtes valeurs de $q / /$, en partie cause par les ondulations à longue portee décrites plus haut, est aussı l'indice que la rugosité ne peut être décrite adéquatement par ce modèle simplifié. Une discussion plus approfondie de cette question sera présentée ailleurs [18].

L'intensité et la position des lobes secondaires varient en fonction de la température de croissance et de la valeur de la désorientation. A titre d'exemple, la Figure 4 montre des cartes d'intensité dans l'espace réciproque dans la direction spéculaire au voisinage du premier pic satellite pour trois échantillons préparés à différentes températures. Les cartes mesurées selon la direction de désorientation ([110]) présentent des lobes latéraux bien définis. Ils sont intenses à $\mathrm{T}=525^{\circ} \mathrm{C}$ mais très faibles à $\mathrm{T}=460^{\circ} \mathrm{C}$. Dans la direction perpendiculaire à la désorientation ([110]), les lobes disparaissent laissant place à des épaulements plus ou moins accentués. L'asymétrie observée dans les les deux plans d'incidence est beacoup plus marquée à forte température. Dans tous les cas, l'espacement des lobes secondaires est d'environ 20-30 fois la largeur attendue des terrasses compte tenu de la désorientation des substrats.

Il est tentant d'expliquer la formation d'une modulation des interfaces dans les super-réseaux $\mathrm{Si} / \mathrm{Ge}$ par le même phénomène d'instabilité morphologique décrit plus haut. Toutefois, plusieurs différences dans la nature et propriétés de la modulation suggèrent une toute autre origine. Dans ce type de structures, des lobes secondaires reliés à une périodicité latérale des interfaces sont observés à des températures de dépôt aussi basses que $100^{\circ} \mathrm{C}$ [19]. Il est improbable qu'une diffusion de surface appréciable soit possible à une telle température. Par ailleurs, ces lobes correspondent à une modulation d'une longueur d'onde de l'ordre de $0.5 \mu \mathrm{m}$ qui n'est pas fonction de la température du dépôt. Enfin, la modulation des interfaces est anisotrope et orientée selon la direction de désorientation du substrat. L'agrégation des marches atomiques présentes à la surface pourrait expliquer l'existence d'un ondulation d'interface dans la direction de désorientation. Le phénomène d'agrégation est possible s'il existe une différence dans la probabilité de liaison d'un adatome sur les marches ascendantes et descendantes. Il est plausible dans le cas d'un système fortement contraint tel que $\mathrm{Si} / \mathrm{Ge}$ qu'une telle différence existe. Par exemple, les atomes de Ge peuvent s'attacher préférentiellement au sommet des marches, sites plus ouverts qui présentent un désaccord de maille moins grand et minimisent la contrainte. Nos résultats suggèrent également qu'il existe une valeur limite -20 à 30- au nombre de marches qui peuvent se regrouper. Un modèle théorique récent [20] prédit une valeur limite au nombre de marches mono-atomiques dans un agrégat. Des travaux additionels sont requis pour confirmer cette interprétation et élucider complètement le phénomène d'ondulation aux interfaces dans les super-réseaux Si/Ge.

\subsection{Suppression de la ségrégation du germanium par l'hydrogène atomique}

La ségrégation de surface est un phénomène courant en épitaxie par jets moléculaires. Les impuretés qui diminuent l'énergie de surface auront tendance à demeurer attachées à la surface plutôt qu'à s'incorporer dans la couche déposée. Sur le silicium, c'est le cas notamment de nombreux dopants communs tels que As, Sb ou B [21]. De la même façon, le profil de concentration d'une couche mince de Ge déposée sur $\mathrm{Si}(001)$ et recouverte d'une couche de Si présente une asymétrie causée par la ségregation de surface. En première approximation, le profil de distribution en profondeur $\mathrm{N}(\mathrm{z}) \mathrm{du} \mathrm{Ge}$ est décrit par une loi exponentielle $N(z)=\frac{N(0)}{\ell} \exp \left(-\frac{z}{\ell}\right)$ où $\ell$ est le coefficient de ségrégation et $N(0)$ est la concentration de surface initiale de Ge. La valeur de $\ell$ varie en fonction des paramètres de croissance et est typiquement de $2 \mathrm{~nm}[21]$.

Il est possible de réduire la ségrégation du Ge en exposant la surface durant le dépôt à une autre espèce atomique dont la ségrégation de surface est plus forte. Certaines impuretés telles que $\mathrm{Sb}, \mathrm{Ga}, \mathrm{Sn}$ peuvent supprimer la ségrégation du Ge. L'incorporation de ces impuretés est toutefọis inévitable à basse température et conduit à un dôpage indésiré du matériau. L'hydrogène sous sa forme atomique réagit fortement avec la surface $(001)$ du Si et peut aussi réduire la ségrégation sans modifier les propriétés électriques du matériau [22]. La Figure 5 illustre l'effet de l'hydrogène sur la distribution de Ge aux interfaces d'un dépôt qui consiste en une série de couches minces de $\mathrm{Ge}$ (4 mono-couches) séparées par des couches épaisses $(40 \mathrm{~nm})$ de Si. Déposées en présence d'hydrogène atomique (zones 2,3 et 4 ), les couches de Ge présentent une distribution plus abruptes qu'en l'absence d'hydrogène (zone 1). La longueur de ségrégation montrée à l'encart de la Figure 5 est de $2 \mathrm{~nm}$ dans les conditions habituelles de dépôt et est réduite à environ $0.5 \mathrm{~nm}$ sous une exposition à $5 \times 10^{-3} \mathrm{~Pa}$ d'hydrogène. A noter toutefois que des expositions plus faibles (zones 3 et 4 ) n'ont qu'un effet mineur sur le profil du Ge. Ces résultats sont en bon accord avec ceux de Ohta et al. [23] obtenus dans une étude similaire.

Nous avons tenté d'appliquer la technique de dépôt sous atmosphère d'hydrogène atomique à la synthèse de super-réseaux $\mathrm{Si} / \mathrm{Ge}$ dans l'espoir d'en améliorer les interfaces. La Figure 6 montre le profil d'intensité du premier pic satellite mesurée en géométrie transversale pour trois super-réseaux préparés -avec et sans la présence d'hydrogène atomique. Dans chaque cas on observe la présence de lobes latéraux 


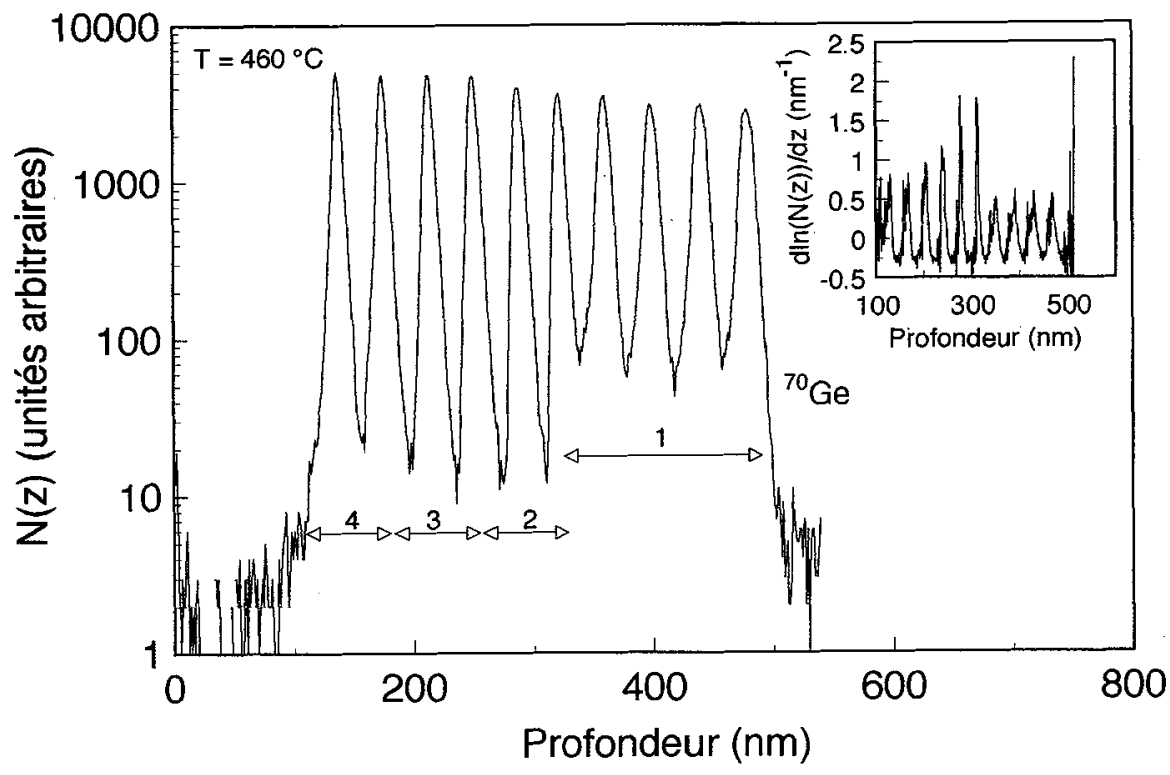

Figure 5: Spectre de masse en ions secondaires de la distribution du ${ }^{70} \mathrm{Ge}$ dans une structure comportant 10 couches minces de Ge (4 mono couches) séparées par des couches de Si de $40 \mathrm{~nm}$ d'épaisseur. Les régions $1,2,3$ et 4 ont été réalisées en opérant la source d'hydrogène atomique à $2140^{\circ} \mathrm{C}$ sous une pression partielle dhydrogène moléculaire de $\sim 0,4 \times 10^{-3}, 1 \times 10^{-3}$ et $2 \times$ $10^{-4} \mathrm{~Pa}$, respectivement. L'encart donne la dérivée logarithmique du signal en fonction de la profondeur.

associés aux ondulations d'interfaces. Ici encore on note une anisotropie reliée à la direction de désorientation des substrats. D'autre part, l'exposition à l'hydrogène réduit de façon significative l'intensité spéculaire indiquant une détérioration globale de la qualité des interfaces. Ceci est probablement causé par une augmentation de la rugosité à courte portée comme en témoigne la forte augmentation de la réflexion diffuse aux grandes valeurs de q// pour les structures déposées en présence d'hydrogène. Ce résultat n'est pas nécéssairement incompatible avec une suppression de la ségrégation de Ge et est probablement relié à une détérioration de la morphologie du dépôt. En effet, une telle dégradation est observée lorsque l'épitaxie est effectuée dans des mauvaises conditions de vide ou en présence d'hydrogène moléculaire [24]. Il est probablement possible de suprimer la ségrégation tout en maintenant une bonne morphologie en exposant la surface à l'hydrogène atomique uniquement lors de la formation des interfaces $\mathrm{Si} / \mathrm{Ge}$. Des travaux en ce sens sont présentement en cours.

\section{CONCLUSION}

Dans ce travail nous avons présenté quelques exemples d'applications des techniques de diffusion des rayons-x à l'étude des interfaces des hétérostructures $\mathrm{Si} / \mathrm{Si}_{1-\mathrm{x}} \mathrm{Ge}_{\mathrm{x}}$. Nous avons pu mettre en évidence des particularités dans la structure des interfaces qui sont reliées aux conditions dans lesquelles le dépôt est réalisé et à la microstructure des substrats utilisés. Ces hétérostructures contraintes présentent une instabilité morphologique qui se développe lorsque l'énergie thermique permet un transport de masse appréciable. Ceci se traduit par une ondulation des interfaces et une modulation latérale de la composition des alliages. Bien qu'indésirables dans l'élaboration de dispositifs, ces effets peuvent néanmoins être exploités dans le développement de nouveaux types de microstructures où les effets de confinement quantiques jouent un rôle de premier plan. On pense ici aux points ou fils quantiques qui deviennent concevables si l'on peut contrôler la morphologie des interfaces. Nos travaux sont encourageants sur ce point car ils montrent qu'il est possible de modifier les interfaces en ajustant les paramètres de dépôt et la structure de surface du substrat. Ce dernier aspect a été mis en évidence de façon particulière dans l'étude 


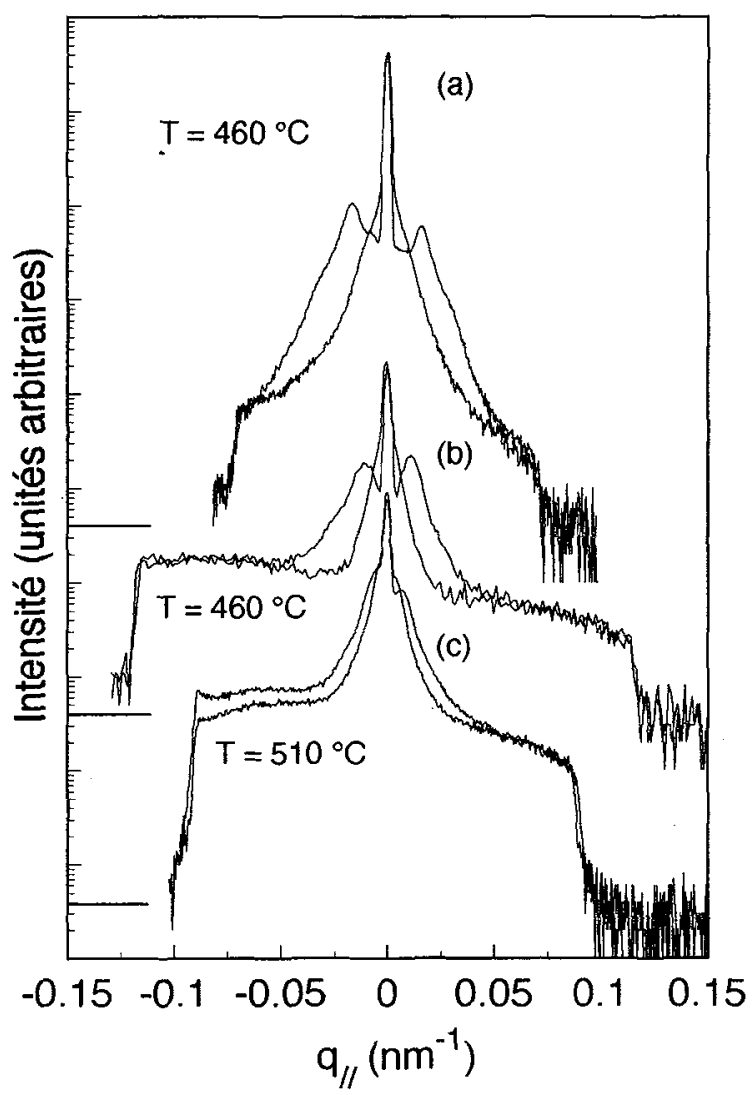

Figure 6: Profil du premier satellite mesuré par un balayage transversal pour trois super-réseaux Si/Ge de 50 périodes déposés (a) dans des conditions normales et (b), (c) déposés en présence d'hydrogène atomique sous une pression partielle de $5 \times 10^{-3}$ $P a$ et à deux températures. Dans chaque cas, on montre les profils mesurés dans la direction parallèle et perpendiculaire à la désorientation du substrat.

des super-réseaux $\mathrm{Si} / \mathrm{Ge}$ de faible périodicité. Pour ces derniers, la désorientation du substrat conduit au développement d'une modulation d'interfaces à longue portée. Il semble possible de contrôler la longueur d'onde et l'orientation de cette modulation en ajustant la vicinalité du substrat et la température de dépôt. Enfin, notre étude de l'influence de l'hydrogène atomique sur l'hétéro-épitaxie $\mathrm{Si} / \mathrm{Ge}$ a montré qu'il était possible de modifier les processus physico-chimiques de surface en cours de dépôt et ainsi améliorer la qualité des interfaces.

\section{Remerciements}

Nous remercions J.P. McCaffrey pour les travaux de microscopie électronique et S.J. Rolfe pour les mesures de spectroscopie de masse des ions secondaires. L'aide de D. Elliot pour l'installation de la source d'hydrogène a été grandement apprécié. 


\section{Références}

[1] voir par exemple Properties of Strained and Relaxed Germanium, E. Kasper, Ed (INSPEC, The Institution of Electrical Engineers, 1995).

[2] Sturm J.C. dans Properties of Strained and Relaxed Germanium, E. Kasper, Ed (INSPEC, The Institution of Electrical Engineers, 1995) pp 193-204.

[3] Baribeau J.-M., Jackman T.E., Houghton D.C., Maigné P., Denhoff M.W., J. Appl. Phys. 63 (1988) 5738-5746.

[4] Baribeau J.-M., Lockwood D.J., Dharma-wardana M.W.C., Rowell N.L. and McCaffrey J.P., Thin Solid Films 183 (1989) 17-24.

[5] Wicks G.W., Rueckwald E.R., Koch M.W. J. Vac. Sci. Technol. B 14 (1996) 2184-2186.

[6] van der Sluis P. J. Appl. Cryst. 27 (1994) 1015-1019.

[7] Parrat L.G., Phys. Rev. 95 (1954) 359-369.

[8] Calculs effectués au moyen du logiciel GIXS de BEDE Scientific.

[9] Asaro R.J., Tiller W.A., Metall. Trans. 3 (1972) 1789-1798.

[10] Grinfeld M.A., Sov. Phys. Dokl. 31 (1986) 831-837.

[11] Cullis A.G., Robbins D.J., Barnett S.J., Pidduck A.J., J. Vac. Sci. Technol. A 12 (1994) 1924-1931.

[12] Ponchet A., Rocher A. Emery J.-Y., Starck C., Goldstein L., J. Appl. Phys. 74 (1993) 3778-3782.

[13] Guyer J.E., Voorhes, P.W., Phys. Rev. Lett. 74 (1995) 4031-4034.

[14] Tersoff J., Phys. Rev. Lett. 76 (1996) 2017-2020.

[15] Tersoff, J., Teichert, C., Lagally, M. G. Phys. Rev. Lett. 76 (1996) 1675-1678.

[16] Baribeau J.-M., J. Phys. D: Appl. Phys. 26 (1993) A156-A160.

[17] Sinha S.K., Sirota E. B., Garoff S., Stanley H.B. Phys. Rev. B 38 (1988) 2297-2311 .

[18] Baribeau J.-M., Lockwood D.J., Labbé H.J., Rolfe S.J., à paraître.

[19] Croce P., Névot L., Rev. Phys. Appl. 11 (1976) 113-125.

[20] Tersoff J., Phang Y.H., Zhang Z., Lagally M.G., Phys. Rev. Lett. 75 (1995) 2730-2733.

[20] Baribeau J.-M., Lockwwod D.J., Syme R.W.G., J. Appl. Phys. 80 (1996) 1450-1459.

[21] Jorke H. dans Properties of Strained and Relaxed Germanium, E. Kasper, Ed (INSPEC, The Institution of Electrical Engineers, 1995) pp 180-189.

[22] Ohtani N., Mokler S.M., Xie H.H., Zhang J., Joyce B.A. "Surface Hydrogen Effexcts on Ge Surface Segregation During Gas Source MBE" dans Int. Conf. Solid State Devices and Materials (Chiba, 29 Aug. - 1 Sept. 1993), pp 249-251.

[23] Ohta G., Fukatsu S., Ebuchi Y., Hattori T., Usami N., Shiraki Y., Appl. Phys. Lett. 65 (1994) 29752977.

[24] Adams D.P., Yasilove S.M., Eaglesham D.J., Appl. Phys. Lett. 63 (1993) 3571-3573. 